\title{
Primary Central Nervous System Lymphoma with Lateral Ventricle Involvement
}

\author{
Yumi Oie ${ }^{1, *}$, Kazuhiro Murayama ${ }^{1}$, Shinya Nagahisa ${ }^{2}$, Masato Abe $^{3}$, Hiroshi Toyama ${ }^{1}$ and Kazuhiro \\ $\mathrm{Katada}^{1}$ \\ ${ }^{1}$ Department of Radiology, Fujita Health University, Japan \\ ${ }^{2}$ Department of Neurosurgery, Fujita Health University, Japan \\ ${ }^{3}$ Department of Pathology, Fujita Health University, Japan
}

\begin{abstract}
A 77-year-old woman presented with worsening cognitive impairment, nausea and vomiting. Computed tomography (CT) of the head revealed a high-density mass that symmetrically filled both lateral ventricles. Magnetic resonance imaging (MRI) showed an enhanced mass with $\mathrm{T} 1$ and $\mathrm{T} 2$ prolongation and restricted diffusion on diffusion weighted imaging (DWI). Diagnosis was delayed until a post-mortem examination, which revealed the mass as a primary central nervous system lymphoma (PCNSL). A high-intensity tumor on DWI with lateral ventricle involvement should raise the possibility of PCNSL.
\end{abstract}

Keywords: Primary central nervous system lymphoma (PCNSL), Magnetic resonance imaging (MRI), Diffusion weighted image (DWI), Magnetic resonance spectroscopy (MRS), Intraventricular tumor.

\section{INTRODUCTION}

Primary central nervous system lymphoma (PCNSL) is a rare type of tumor, accounting for $5 \%$ of all intracranial neoplasms [1]. The most common localization sites of PCNSL are in the supratentorial white matter of the frontal lobes [1-3]. Diagnosis of PCNSL is often difficult due to variability in their imaging patterns and locations. Here we report a case of an unusual PCNSL with lateral ventricle involvement.

\section{CASE REPORT}

A 77-year-old woman was admitted to hospital with a 4month history of cognitive impairment and a 3-month history of nausea and vomiting. Lactate dehydrogenase (LDH), soluble interleukin receptor (sIL-2R), and tumor marker levels were all within normal limits. Cerebrospinal fluid (CSF) findings showed a cell count of 54/3 (normal: 0/3$15 / 3, / \mathrm{mm}^{3}$ ), total protein $199 \mathrm{mg} / \mathrm{dl}$ (normal: $10-40 \mathrm{mg} / \mathrm{dl}$ ), and glucose $49 \mathrm{mg} / \mathrm{dl}$ (normal: $50-75 \mathrm{mg} / \mathrm{dl}$ ). Neuropsychological examination revealed mild dementia (mini-mental state examination (MMSE): 23). Computed tomography (CT) revealed a high-density mass that symmetrically filled both lateral ventricles. Physiologic calcifications of the choroid plexus were found at the bilateral margins of the mass, with no signs of calcification in the mass (Fig. 1). Magnetic resonance imaging (MRI) showed a solid, enhancing mass extending from the bilateral ventricles to the fourth ventricle. T2-weighted images showed an iso-intense mass that exhibited areas of low

*Address correspondence to this author at the 1-98, Dengakugakubo, Kutsukake, Toyoake, Aichi 470-1192, Department of Radiology, Fujita Health University, Japan; Tel: 81-562-93-9259; Fax:81-562-95-2253;

E-mail: yumi-oie@fujita-hu.ac.jp intensity on T1-weighted images . Furthermore, the mass showed high-intensity on DWI and low signal intensity on the apparent diffusion coefficient (ADC) map (Fig. 1, 2). Proton magnetic resonance spectroscopy (MRS) was performed with a $1.5 \mathrm{~T}$ whole-body-system and revealed a high lipid peak, decreased $\mathrm{N}$-acetylaspartate and increased choline but no lactate peak (Fig. 3). ${ }^{123}$ I-IMP brain perfusion single-photon emission computed tomography (SPECT) showed mild tumor uptake in both lateral ventricles.

A biopsy was recommended to the patient and her family, but was refused. Three months later, the patient experienced a disturbance of consciousness. A follow-up CT revealed a significant increase in the size of the mass, compared with the initial presentation (Fig. 4). The patient died 11 days later, and an autopsy was performed. The autopsy revealed that tumor mass extended from both lateral ventricles to the fourth ventricle (Fig. 5), consistent with image findings (Fig. 2) showing brain herniation. The extracranial organs were normal in appearance with no evidence of malignancy elsewhere. Microscopically, tumor cells had infiltrated into the Virchow-Robin space and parenchyma (including the medulla oblongata, hippocampus and corpus callosum). As tumor cells were found in the walls of the ventricles and choroid plexus, it was postulated that the mass originated within the ependymal or subependymal vascular space. Histologically, the mass contained a monomorphic proliferation of large lymphoid cells. Immunohistochemical staining revealed expression of CD20 and MUM1 (Fig. 6). These findings confirmed a high-grade, large B-cell, PCNSL.

\section{DISCUSSION}

PCNSLs are rare tumors accounting for 5\% of all intracranial tumors $[4,5]$. 


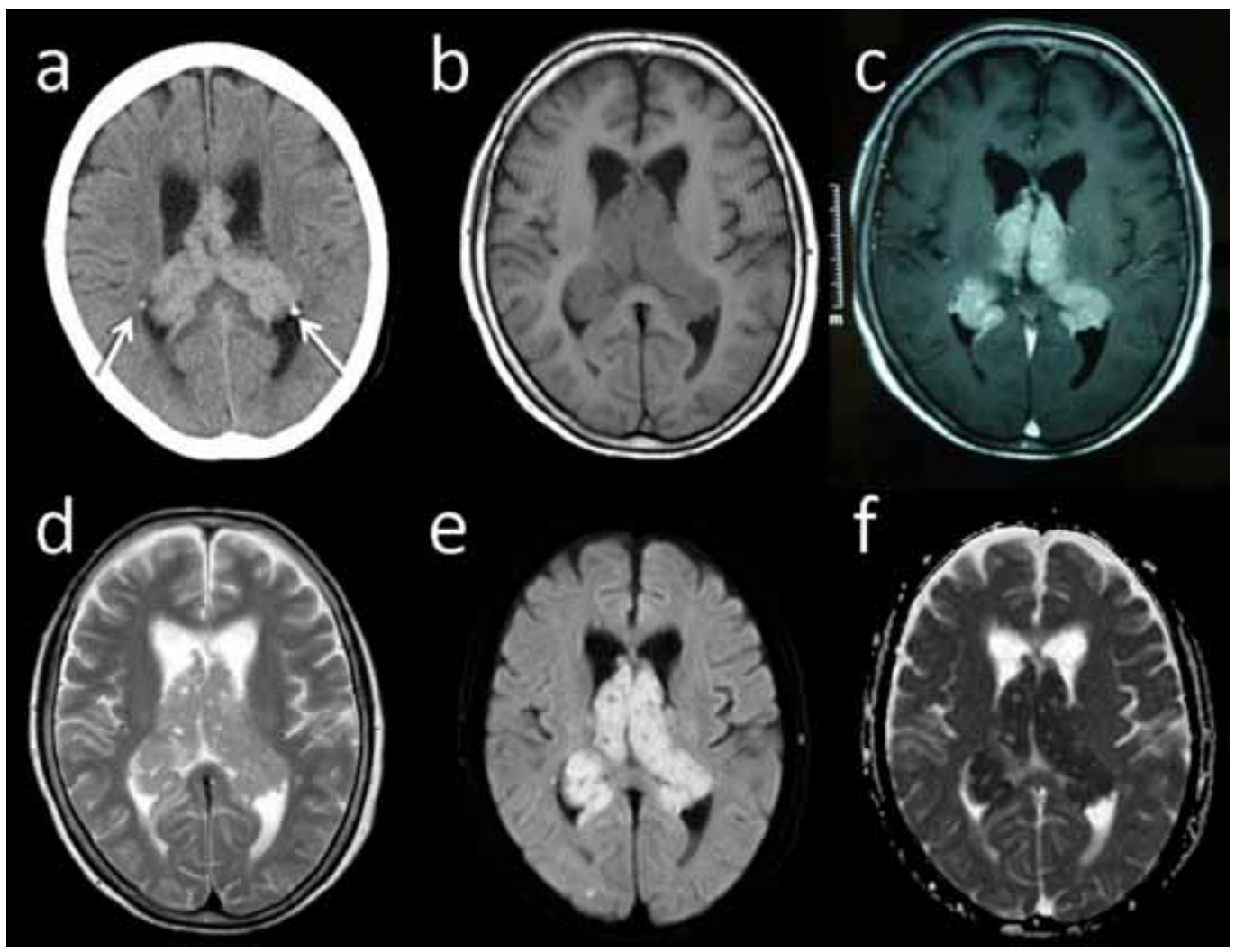

Fig. (1). The tumor symmetrically fills both lateral ventricles. Brain computed tomography (CT) (a) shows a high-density mass in both lateral ventricles. Physiologic calcifications of the choroid plexus are found at the bilateral margins of the mass (arrows), but no calcification is found in the mass. T1-weighted magnetic resonance imaging (MRI) (b) shows a low intensity mass with marked enhancement (c). T2weighted MRI shows an isointense mass lesion (d). Diffusion weighted imaging (DWI) shows the tumor with high signal intensity with restricted diffusion (e) with corresponding hypointensity on the apparent diffusion coefficient (ADC) map (f).
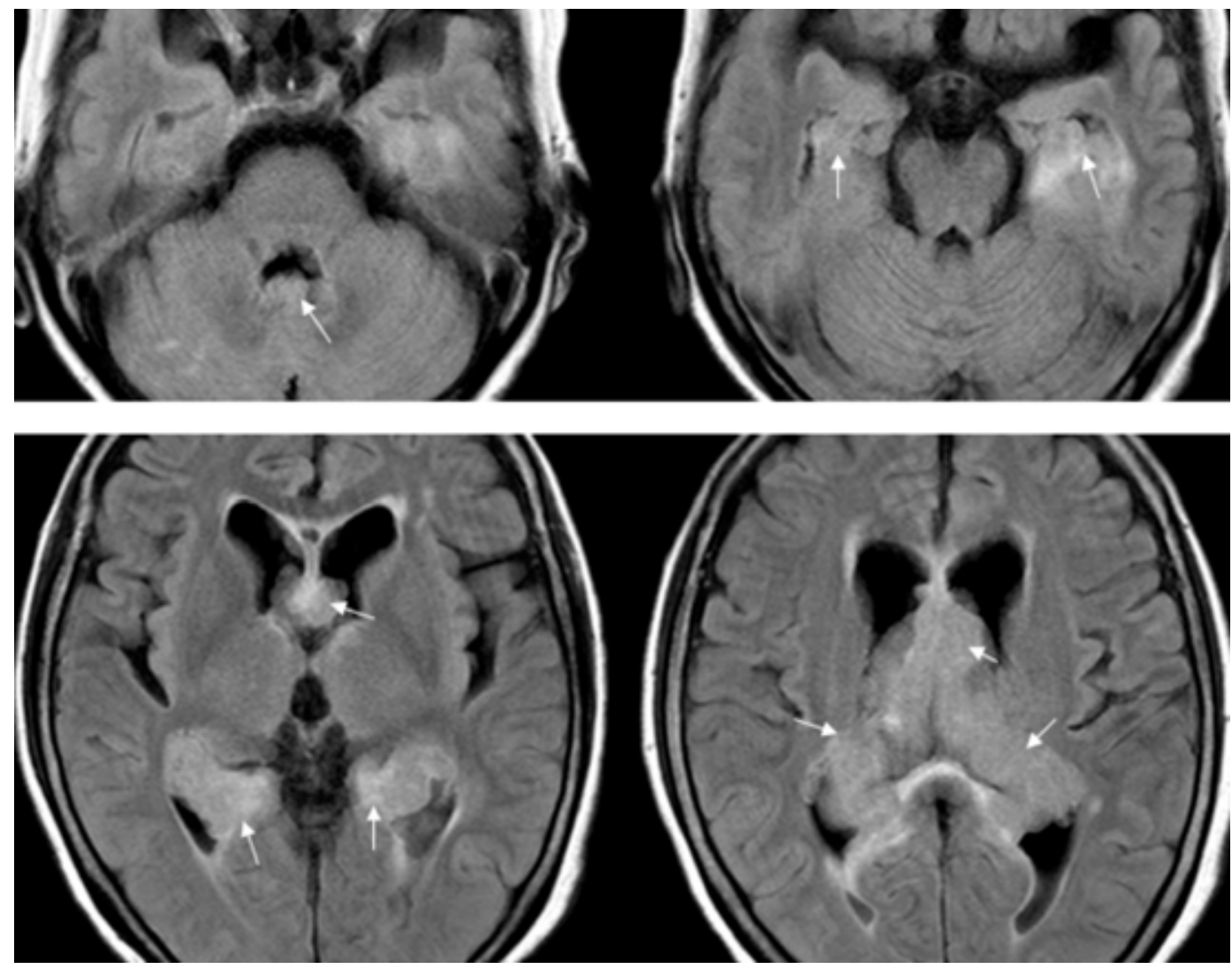

Fig. (2). FLAIR shows the high-intensity tumor extends from both lateral ventricles to the fourth ventricle. 

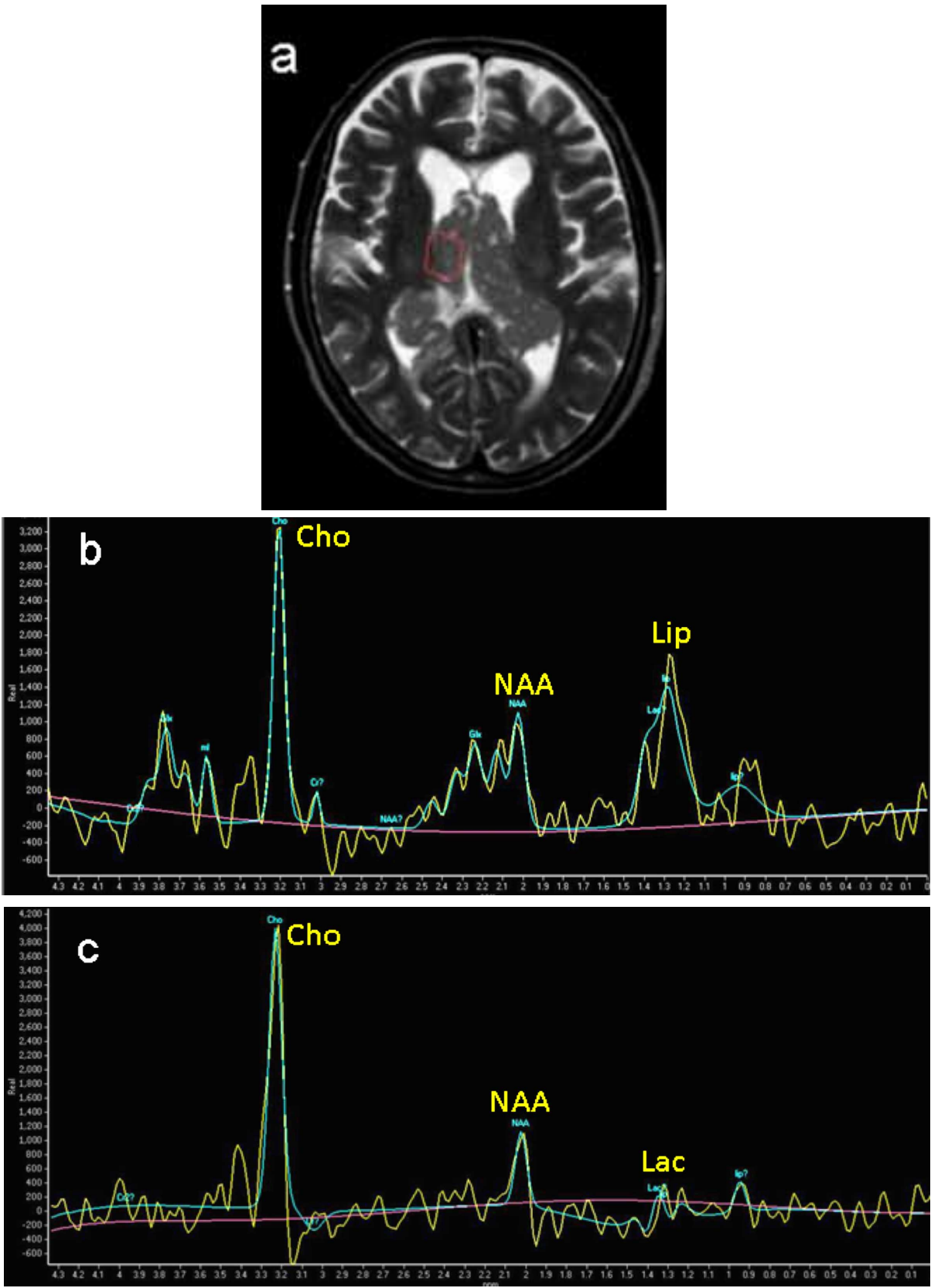

Fig. (3). Proton MRS in PCNSL: (a) This T2-weighted image shows the location where the spectrum was acquired (square line). (b) TE 34 ms. (c) TE $144 \mathrm{~ms}$. TR is fixed at 2000ms. A high lipid (Lip) peak is recognized. A lactate (Lac) peak is not visible. Choline (Cho) peak is increased and N-acetylaspartate (NAA) peak is decreased.

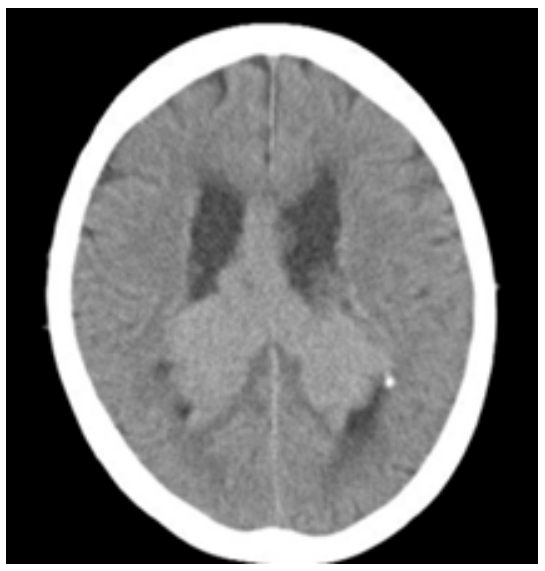

Fig. (4). Brain CT imaging at the time of second hospital admission shows the enlarging tumor. 


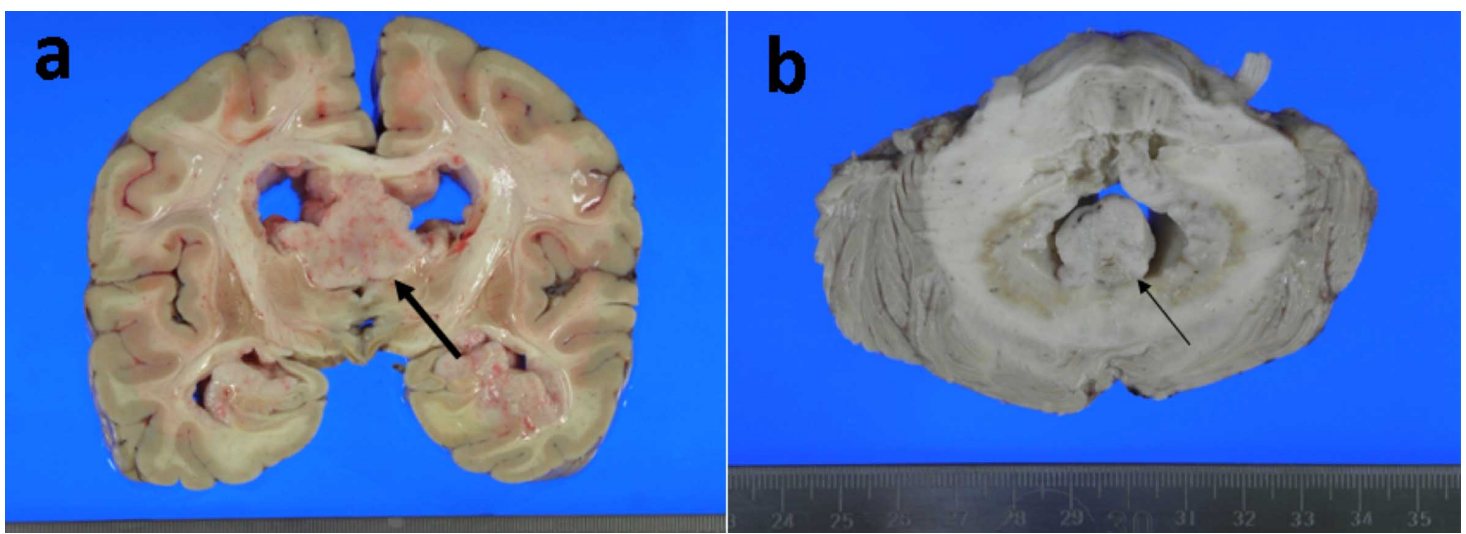

Fig. (5). (a) Brain coronal section shows a mass in both lateral ventricles, as shown in the imaging findings on Fig. 2. (b) An axial section at the level of the fourth ventricle also demonstrates the mass.
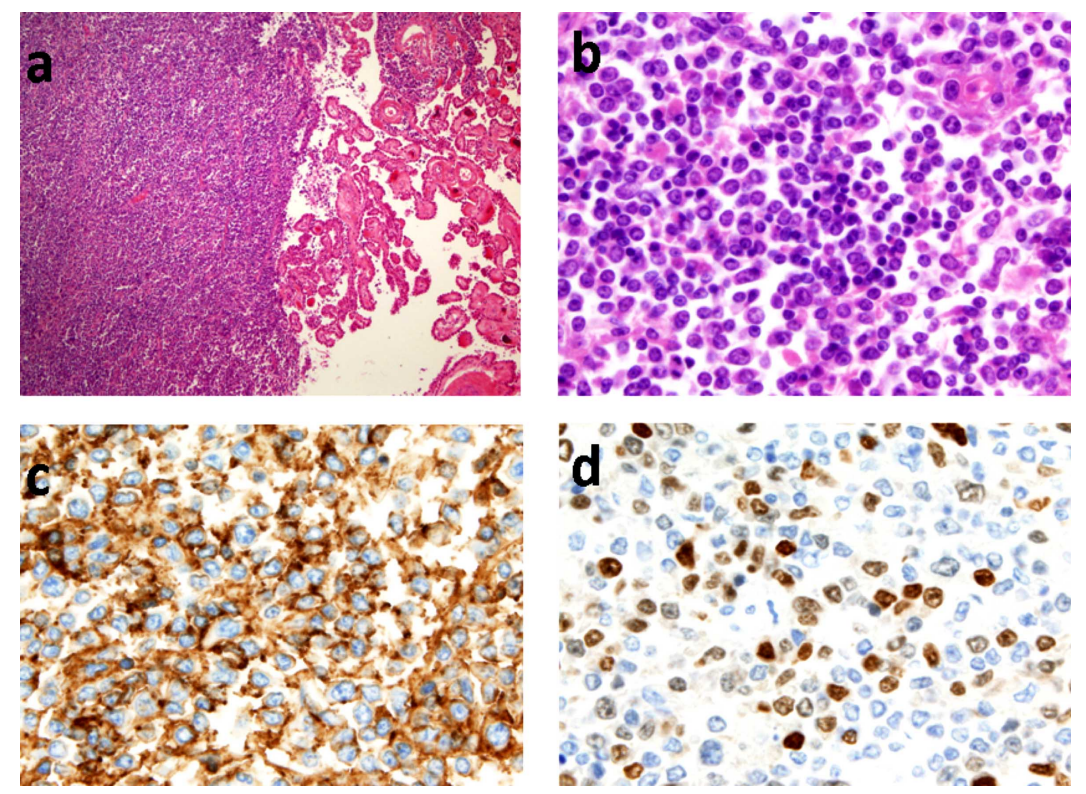

Fig. (6). Histological examination shows (a) tumor cell expression between the wall of the ventricles and choroid plexus (H\&E). (b) Diffuse proliferation of large cells with nuclei (H\&E). Immunohistochemical staining reveals expression of CD20 (c) and MUM1 (d).

PCNSL presents with a variety of focal or non-focal signs and symptoms. The clinical presentation is characterized by neurologic deficits, mental status changes, seizures, and increased intracranial pressure. When psychiatric symptoms are prominent it is difficult to distinguish PCNSL from other brain disorders that cause cognitive impairment [5].

PCNSL typically appear on CT as high or iso-dense, hypo- or iso-intense on unenhanced T1-weighted MR, and iso- or high-intense on T2-weighted MR images. Most lesions show moderate-to-marked contrast enhancement, while calcification and necrosis are rare. As PCNSL are highly cellular tumors, water diffusion is often restricted, making them appear with a high-intensity on DWI images and a low intensity on ADC maps. Proton MRS presents with high $\mathrm{Cho/Cr}$ ratios and elevated lipid peaks [6].

The differential diagnosis for the imaging findings of an intraventricular mass includes central neurocytoma, ependymoma, colloid cyst, choroid plexus papilloma, metastasis, or high-grade glioma [7]. As the lesion was characterized by symmetrical filling of the ventricles, highintensity on DWI and a well-enhanced solid mass, we felt that the most likely the diagnosis was lymphoma, metastasis, or high-grade glioma. In this case the tumor mass presented with a typical signal pattern for lymphoma on MRI; however, the location was very unusual. PCNSL occur in various regions of the brain, most commonly in the cerebral hemisphere $(31.4-44 \%)$ [2, 3, 8]. However, lesions also appear in the basal ganglia and thalamus (15.5-24\%), corpus callosum $(5-15.7 \%)$, ventricles $(7-11.8 \%)$, brain stem (2.4$8 \%)$, cerebellum $(9.4-14.3 \%)$, spinal cord $(1.2-1.4 \%)$ and the eye $(2.9-4.1 \%)[2,3,8]$. Previous studies reported the diagnostic utility of several tests for the diagnosis of PCNSL. Because PCNSL are highly cellular tumors, water diffusion is often restricted making them appear high-intensity on DWI and hypo-intensity on ADC map, consistent with our case [9]. Shinoda et al. showed the high ${ }^{123}$ I-IMP retention on SPECT image was characteristic in PCNSL [10]. However, mild uptake of ${ }^{123}$ I-IMP by the tumor was not a useful marker for diagnosis of PCNSL in our case. Although serum sIL-2R are generally useful marker for systemic lymphoma patients, Kitai et al. reported the difference of serum IL-2R between PCNSL and other brain diseases was not significant such as in our case [11]. 
In summary, a PCNSL with ventricle involvement confirmed by autopsy has been presented. PCNSL is a treatable neoplasm, although a biopsy is required for diagnosis. A high-intensity tumor on DWI with lateral ventricle involvement raises the possibility of PCNSL.

\section{CONFLICT OF INTEREST}

The authors confirm that this article content has no conflicts of interest.

\section{ACKNOWLEDGEMENT}

Declared none.

\section{REFERENCES}

[1] Küker W, Nägele T, Korfel A, et al. Primary central nervous system lymphomas (PCNSL):MRI features at presentation in 100 patients. J Neurooncol 2005; 72: 169-77.

[2] Haldorsen IS, Krakenes J, Krossnes BK, et al. CT and MR imaging features of primary central nervous system lymphoma in Norway, 1989-2003. AJNR Am J Neuroradiol 2009; 30: 744-51

[3] Johnson BA, Fram EK, Johnson PC, et al. The variable MR appearance of primary lymphoma of the central nervous system: comparison with histopathologic features. AJNR Am J Neuroradiol 1997; 18: 563-72.
[4] van der Sanden GA, Schouten LJ, van Dijck JA, et al.; Working group of specialists in neuro-oncology in the Southern and Eastern Netherlands. Primary central nervous system lymphomas: incidence and survival in the Southern and Eastern Netherlands. Cancer 2002; 94: 1548-56.

[5] Haldorsen IS, Krossnes BK, Aarseth JH, et al. Increasing incidence and continued dismal outcome of primary central nervous system lymphoma in Norway 1989-2003: time trends in a 15-year national survey. Cancer 2007; 110: 1803-14.

[6] Haldorsen IS, Espeland A, Larsson EM. Central nervous system lymphoma: characteristic findings on traditional and advanced imaging. AJNR Am J Neuroradiol 2011; 32: 984-92.

[7] Jelinek J, Smirniotopoulos JG, Parisi JE, et al. Lateral ventricular neoplasms of the brain: differential diagnosis based on clinical, CT, and MR findings. AJR Am J Roentgenol 1990; 155: 365-72.

[8] Bühring U, Herrlinger U, Krings T, et al. MRI features of primary central nervous system lymphomas at presentation. Neurology 2001; 57: 393-6.

[9] Stadnik TW, Chaskis C, Michotte A, et al. Diffusion-weighted MR imaging of intracerebral masses: comparison with conventional MR imaging and histologic findings. AJNR Am J Neuroradiol 2001; 22: 969-76.

[10] Shinoda J, Yano H, Murase S, Yoshimura S, et al. High ${ }^{123}$ I-IMP retention on SPECT image in primary central nervous system lymphoma. J Neurooncol 2003; 61: 261-5.

[11] Kitai R, Sasaki H, Matsuda K, et al. Measurement and cellular sources of the soluble interleukin-2 receptor in primary central nervous system lymphoma. Brain Tumor Pathol 2012. [Epub ahead of print]

(c) Oie et al.; Licensee Bentham Open.

This is an open access article licensed under the terms of the Creative Commons Attribution Non-Commercial License (http://creativecommons.org/licenses/bync/3.0/), which permits unrestricted, non-commercial use, distribution and reproduction in any medium, provided the work is properly cited. 